\title{
A Classification Framework for Context-aware Mobile Learning Systems
}

\author{
Richard A.W. Tortorella \\ School of Computing, University of Eastern Finland, Joensuu, FIN-80101, Finland \\ Email: tortorella@ieee.org \\ Kinshuk \\ College of Information, University of North Texas, Denton, 76203-5017, USA \\ Email:kinshuk@ieee.org \\ Nian-Shing Chen \\ National Sun Yat-sen University, Kaohsiung, 80424, Taiwan \\ Email: nschen@mis.nsysu.edu.tw \\ Sabine Graf \\ School of Computing and Information Systems, Athabasca University, Edmonton, T5J3S8, Canada \\ Email: sabineg@athabascau.ca
}

\begin{abstract}
The field of context awareness is ever increasing due to the proliferation and omnipresent nature of mobile computing devices. Not only is learning becoming ubiquitous, but the sensors in mobile devices are permitting learning systems to adapt to the context of the learners. This paper provides a classification framework for the field of context-aware mobile learning, which is applied to papers published within selected journals from January 2009 to December 2015 inclusive. Obtained from the combined fields of context awareness and educational technology, a total of 2,968 papers are reviewed, resulting in 41 papers being selected for inclusion in this study. The classification framework consists of three layers: hardware architecture layer, context architecture layer and an evaluation layer. The framework will allow researchers and practitioners to quickly and accurately summarize the status of the current field of context-aware mobile learning. Furthermore, it has the potential to aid in future system development and decision making processes by showing the direction of the field as well as viable existing methods of system design and implementation.
\end{abstract}

Index Terms-Context-aware learning system, mobile learning, adaptive learning, classification framework, ubiquitous learning, context-awareness

\section{INTRODUCTION}

Mobile information and communication technologies have become common in recent years, particularly because of smartphones, where the proliferation of the technology has allowed users to utilize applications anywhere at any time [1]. This prevalence of smartphones has caused learning to change, as educational technology developments and the availability of digital content has led to a change in traditional teaching and interactions $[1,2]$. The ubiquitous availability of mobile devices has promoted a seamless way of learning that advocates the merging of learning into everyday life [3], therefore allowing mobile learning to itself become ubiquitous.

Current advances in smartphone technology have also resulted in the micro-miniaturization of sensors present in mobile devices. These sensors have become diverse and pervasive and are adapted for a myriad of different uses [4]. Over twenty years ago, researchers had started to design systems that reacted and adapted to an individual's changing context [5]. This adaptation to context is key to the design of adaptive mobile learning systems [6].

One of the precursors, and a field that set the stage for context-aware mobile learning was ubiquitous computing. Ubiquitous computing was described in the late 1980s as the type of computing found in a world that is interwoven with sensors and computational elements which are embedded seamlessly into everyday objects [7]. Ubiquitous computing laid the ground work for ubiquitous learning, a new educational paradigm, made possible in part by digital media, which focuses on the needs and dynamics of learning [8]. In turn, ubiquitous learning led to context-aware ubiquitous learning: an innovative approach that integrates context-aware technologies in order to detect and adapt to, the context and situation of learners in the real world [9].

Today, context-aware mobile learning is growing in its importance due to the continually changing context in which the learners find themselves [10]. With the increased attention of researchers in the field, the rate of the literature published on mobile and ubiquitous learning has been steadily increasing as well [11]. For 
example, from 2005 to 2010, the number of published papers is four times that of the previous five years [11]. With the current advent of technology, this trend is likely to continue at the same rate of growth in coming years.

The aim of this research is to identify the system designing trends present within context-aware mobile learning systems from the literature published in 2009 to 2015. Furthermore, a classification framework is also proposed to allow for subsequent integration and classification of additional literature that is emerging past the 2015 date. This paper is therefore divided into two main sections. The next section describes the method of analysis of the literature selection process for this research. This is followed by the description of the framework consisting of the various trends and classifications which summarize various of aspects of context-aware mobile learning systems.

\section{RELATED WORK}

While there has been limited work directly addressing the state of the research in context-aware mobile learning, various studies have provided insights into the larger fields of context awareness and mobile learning. In a well cited study involving context-aware systems, Baldauf, Dustdar, \& Rosenberg [12] discuss the various types of context-aware architectural design principles and derive a design framework to explain the different elements common to most context-aware architecture. Their paper provides a very good starting point for modern research into the field of context-aware computing, and the resulting frameworks. In 2009, Hong, Suh and Kim [13] published a review of context-aware systems from 2000 to 2007. The outcome of the review was a framework based on the system architecture of context-aware systems, with the focus on the physical hardware and application layers. A more recent survey of contextaware computing was published by Perera, Zaslavsky, Christen and Georgakopoulos in 2014 [14]. Although helpful in understanding the trends of the aspects of context-aware systems, the focus was on a subset of 50 commercial solutions proposed in the field of contextaware computing. The current literature, although valuable in shedding further light onto the field of context-aware mobile learning, does not directly address learning systems, and does not address more recent changes seen within the field. It is therefore hoped that more recent studies involving trends in closely related areas may serve to bring some insights into the adaptive learning aspect and the current status of the field of context-aware mobile learning.

Similar to context-aware mobile learning, another related computing field involving learning is pervasive learning, which focuses on the detection of information in the context of the learner [1]. In 2014, Lucke and Rensing provided a comprehensive qualitative overview of the existing work in the field of pervasive learning. They showed that there was still a very high demand for further research and development within the field [1]. Another closely related field involving context-aware systems is that of augmented reality. According to $\mathrm{Wu}$, Lee, Chang, \& Liang [15], augmented reality systems typically have three features: a combination of real and virtual worlds, real time interaction and an accurate 3D registration of virtual and real objects. It is the middle aspect, real time interaction, which applies to contextaware systems, as in both cases the system adapts to real world events or conditions. However, although the authors discuss issues and challenges faced by the field, the article neither provides details about the current state of the field nor the direction in terms of system design and details appertaining to learning [15]. In 2011, Hwang and Tsai described the research trends found in mobile and ubiquitous learning from 2001 to 2010 [11]. Three aspects of the field were reviewed: the status of the field (increasing or decreasing), sample groups and learning domains.

Building on, and following in the steps of previous works, this paper provides a comprehensive review of trends in context-aware mobile learning systems from 2009 to 2015. Beyond the existing literature such as Hwang and $\mathrm{Wu}$ [16], this paper further increases the focus beyond the wide breadth of mobile technologyenhanced learning, and narrows its gaze upon the key aspect of learning within context-aware systems. This learning aspect focuses on the type of learning that takes place, the subject matter and the means by which context adaptation is achieved. A resulting framework emerges from this study comprised of the hardware architecture layer, context determination layer and evaluation layer. The proposed framework would be useful to researchers and practitioners by providing a common means of detecting and reviewing trends in the existing literature. Therefore, this framework could serve as a guide in providing an insight into the future of mobile learning.

In order to be able to perform a comprehensive overview of the field of context-aware mobile learning, the literature in the field must be identified and then distilled into a means of understanding the subject matter. To achieve this, the top twenty journals were selected from the fields of context awareness and educational technology. Journals were specifically targeted as they contain high quality papers that have a greater chance of having completed in-depth comprehensive studies on their presented subject matter. From these top twenty journals, those papers were selected which satisfied the criteria defining them as related to context-aware mobile learning. The following section explains the detailed paper review process, including the journal selection process and paper selection process, including selection criteria. The subsequent section describes the resulting framework and analysis of the reviewed literature.

\section{JOURNAL AND PAPER SELECTION}

In order to ensure that trends in context-aware mobile learning were properly identified, a list of journals was required in order to perform a search for papers appertaining to the subject matter. As there is no specific research field that covers context-aware mobile learning 
directly represented in computing science, two parent research fields were identified whose common elements would adequately incorporate the required field. These two research fields are context awareness and educational technology. These two fields were selected as their merger /overlap defined the field of context-aware mobile learning. From these two fields, Google Scholar was used to select the top 10 journals from each field using the journals' ranking via their h5-index score.

Once the journals were selected, papers would need to be identified from those journals which describe the field of context-aware mobile learning. The authors decided that seven keywords listed would be the best representation of the research field of context awareness, and nine for the research field of educational technology (Table 1).

Table 1. Keyword Selection

\begin{tabular}{|l|l|}
\hline $\begin{array}{l}\text { Educational Technology } \\
\text { Keywords }\end{array}$ & $\begin{array}{l}\text { Context Awareness } \\
\text { Keywords }\end{array}$ \\
\hline School & Context \\
\hline Teach & Location \\
\hline Education & Mobile \\
\hline Learn & Pervasive \\
\hline Instruction & Position \\
\hline Training & Sensors \\
\hline Curriculum & Ubiquitous \\
\hline Academic & \\
\hline Student & \\
\hline
\end{tabular}

Each of the journals was searched for papers which contained one or more keywords from each column in either their title or abstract. From the thousands of possible candidates containing the keywords, there were 41 remaining papers which involved context-aware mobile learning. These papers will be reviewing using the following classification framework.

\section{ClASSIFICATION FRAMEWORK}

With the appropriate papers identified by the selection process, the task of content analysis was next. Two of the key tenets to any literature review are the organization of the existing literature, and the evaluation of the literature in terms of their individual differences and similarities to one another. This paper defined the field of contextaware mobile learning as the merger of two larger disciples: context awareness and educational technology. Therefore, the selected means of organizing and evaluating the existing literature must also reflect those unique research fields, resulting in a framework.

In order to classify and evaluate trends related to the context awareness field of context-aware mobile learning, the context adaptation of the system needs to be investigated. Therefore, one of the layers of the framework was created directly addressing context adaptation: the context determination layer. This layer addresses the type of context towards which the system being reviewed is adapting. Additionally, the type of sensor and any further adaptivity that may take place are analyzed.

However, in order to properly address the context adaptation of the system, the hardware itself must also be reviewed, as it is a key aspect to the means of adaptation. To this end, the hardware architecture layer was created. In this layer, the type of device being used, the system infrastructure and the connection types used by the various systems are reviewed. As the primary focus of the hardware layer is on how the hardware interacts with the context layers, aspects such as user interface, hardware performance and hardware details are not addressed.

The second parent research field of context-aware mobile learning, is that of the educational technology. Therefore, an evaluation layer was created in order to address education related aspects of the various systems being reviewed. These include the means by which the system itself was evaluated during the study, the duration of testing, participant age and number as well as the subject matter being taught.

This section presents a framework presented in Table 2 containing the above described layers. They are created in such a manner in order to classify and summarize trends within the context-aware mobile learning field.

The following presented results were analyzed by applying the above proposed classification framework on the literature obtained from the paper review process.

\section{A. Hardware architecture layer}

The type of hardware involved in the various papers is key to understanding the direction of the research as a whole. With increases and advances in key hardware components found in this field, the field will also benefit and advance. Therefore it is imperative that the hardware in question is understood and evaluated. To this end, the hardware architecture layer of the framework focused on the following aspects:

- Device Used - Type of physical device used in various studies.

- System Infrastructure - Server based or standalone infrastructure employed in the various studies.

- Connection Type - The means by which the device connected to the system infrastructure.

Additionally, trends over time involving the hardware used by the various studies were reviewed in order to further shed light on the subject matter.

\section{$\underline{\text { Device used }}$}

This section describes the type of physical device used by the learners in various studies, which does differ between papers. As can be seen in Fig. 1, the most common type of device being used is surprisingly the Personal Digital/Data Assistant (PDA) at 20 entries, or at $49 \%$ of the devices. 
Table 2. The classification framework

\begin{tabular}{|c|c|c|c|c|}
\hline Layer & \multicolumn{4}{|c|}{ Classified Category } \\
\hline $\begin{array}{l}\text { Hardware } \\
\text { Architecture } \\
\text { Layer }\end{array}$ & $\begin{array}{ll} & \text { Device used } \\
\text { - } & \text { PDA } \\
\text { - } & \text { Smartphone/Mobile } \\
\text { - } & \text { Tablet } \\
\text { - } & \text { Handheld }\end{array}$ & $\begin{array}{ll} & \text { System infrastructure } \\
\text { - } & \text { Standalone } \\
\text { - } & \text { Server based }\end{array}$ & $\begin{array}{l}\text { Connection type } \\
\text { - } \quad \text { Wireless (Wi- } \\
\text { Fi) } \\
\text { - } \quad \text { Mobile/Cellular }\end{array}$ & \\
\hline $\begin{array}{l}\text { Context } \\
\text { Determinatio } \\
\text { n Layer }\end{array}$ & $\begin{array}{l}\text { Type of context } \\
\text { - } \quad \text { Ambient: temperature, humidity } \\
\text { - } \quad \text { Location } \\
\text { Movement: } \\
\circ \quad \text { Movement of device } \\
\circ \quad \text { Movement of user } \\
\text { - Temporal }\end{array}$ & $\begin{array}{ll} & \text { Type of sensor } \\
& \\
\text { - } & \text { Accelerometer } \\
\text { Global positioning } \\
\text { system (GPS) } \\
\text { - } & \text { Radio frequency } \\
\text { identification } \\
\text { (RFID) }\end{array}$ & & \\
\hline $\begin{array}{l}\text { Evaluation } \\
\text { Layer }\end{array}$ & $\begin{array}{ll} & \text { System evaluation } \\
\text { - } & \text { Survey/questionnaire } \\
\text { - } & \text { Pre-post tests } \\
\text { - } & \text { Interview } \\
\text { Other }\end{array}$ & $\begin{array}{ll} & \text { Duration of testing } \\
\text { - } & \text { Full day } \\
\text { - } & \text { Full week } \\
\text { - } & \text { Part day } \\
\text { - } & \text { Part week } \\
\text { - } & \text { Part month }\end{array}$ & $\begin{array}{ll} & \text { Participant } \\
\text { - } & \text { Number of } \\
\text { - } & \text { participants } \\
\text { Participant age }\end{array}$ & $\begin{array}{l}\text { Subject matter } \\
\text { - } \quad \text { Learning } \\
\text { type } \\
\text { - } \quad \text { Discipline }\end{array}$ \\
\hline
\end{tabular}

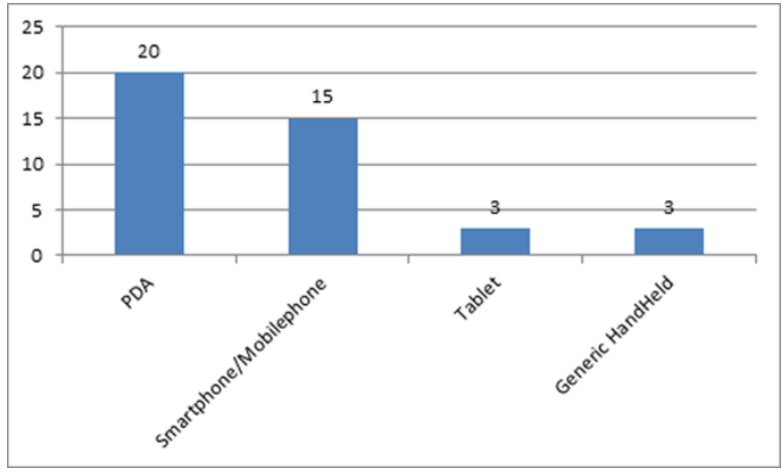

Fig.1.Type of Device Being Used (Number of Papers)

The large number, and first place of PDAs present within studies is surprising as the PDA has certainly declined in usage over the last few years. As can be seen from Fig. 2, although the usage of PDAs decreased over time, this occurred much later than would be expected to follow their general popularity.

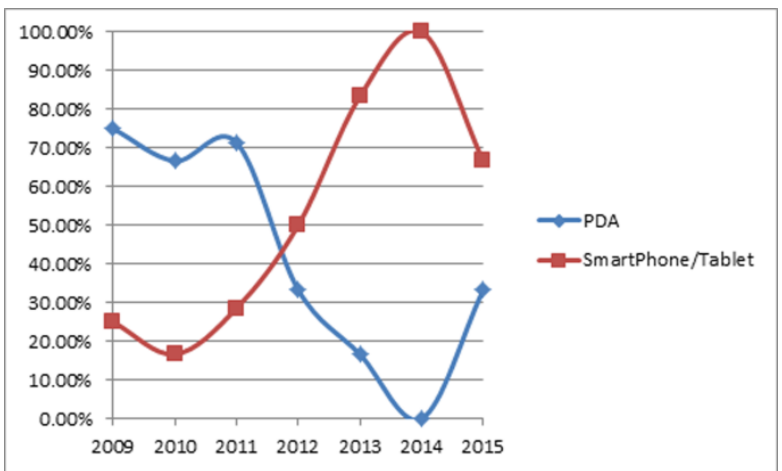

Fig.2. PDA vs. Smartphone/Tablet Usage (Percent of Papers Published)
The suspected reason for the continued prevalence and longevity of the PDA is twofold:

1. Given the age of the papers examined (from 2009 to 2015) there were certainly more PDAs available in the first half and may account for the higher than expected numbers.

2. As will be seen in Fig. 7, the most common type of sensor used for context-aware adaptation are Radio Frequency Identification (RFID) tags. PDAs represent one of the few platforms that enable the user to insert or add an additional sensor into the existing Compact Flash (CF) slot available on most late models. This expandability is not as straightforward with other devices which may explain why PDA remained a popular choice (Fig. 2).

The unexpected dominance of the PDA as a device type may be driven not only by cost (PDAs are generally cheaper than smartphones which ranked second at 15 or $37 \%$ ) but the availability of expansion slots not commonly found on smartphones/mobile phones.

The other two types of devices are the tablet (such as the iPad), and those where no specific hardware was mentioned save the term mobile handheld device.

\section{System infrastructure}

The two types of system infrastructure that were commonly used were server based (mentioned in 31 papers), and standalone systems (mentioned in 8 papers). Server based systems utilized a central server (independent of the device) to act either as a repository for information or for offsite hardware support for computational processing. On the other hand, standalone 
systems, as the name suggests, were self-contained systems on learners' devices with no need for external support.

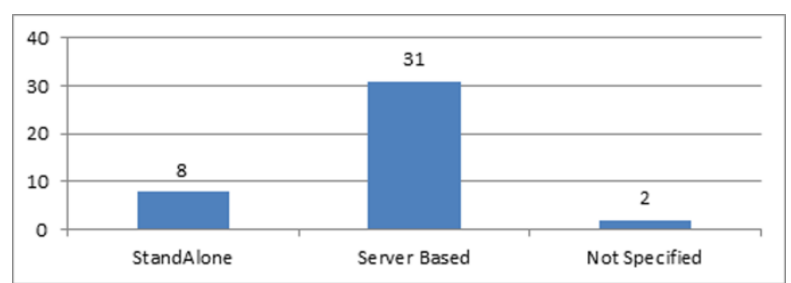

Fig.3. System Infrastructure (Number of Papers)

Fig. 3 shows that server based infrastructure was four times as common as standalone units: there are a few possible reasons for this. Firstly, as shown in Fig 1., the most common type of hardware for the years of 2009 to 2015 inclusive has been the PDA. These devices were not known for having large amounts of memory or computational power, and could use a server to provide these services. Secondly, if more than one concurrent learner is involved in the study (increasing likelihood with the proliferation of smartphones), it could be argued that having a central storage for information dissemination would be better than having to update multiple (in some studies hundreds) devices.

\section{Connection type}

When the system did need to communicate with a server (in a server based setup) or additional infrastructure, the question of what type of connection has been utilized needs to be determined in order to properly understand the overall system architecture.

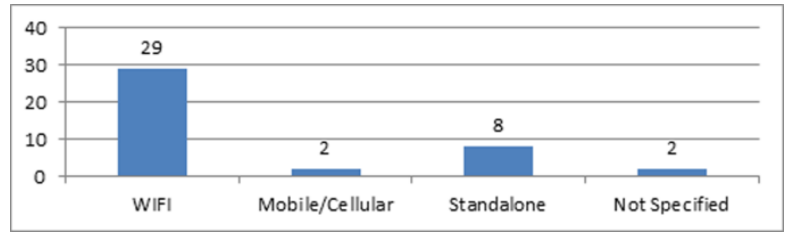

Fig.4. Hardware Connection Type (Number of Papers)

In line with the type of device infrastructure being used, Fig 4. shows that 8 standalone devices or $20 \%$ of the total used no type of data connection. The remaining connections were a wireless Wi-Fi connection at a staggering 29 or $94 \%$ of the devices with a network connection, with the exception of one using the mobile/cellular network. This can be due to the ubiquitous nature of wireless 802.11 connections and the lack of cost associated with a Wi-Fi connection over a cellular connection for data transfer. However, cellular connections are appropriate in locations where a Wi-Fi signal may not be present. An example of this would be the study conducted by [17], where smartphones were used around the town of Barcelona in Spain, where a WiFi connection would not have been the best option.

\section{B. Context determination layer}

The ability to classify and detect trends in the type of context involved in various studies is fundamental to understanding the future direction and trends of the field. The focus of the context determination layer is to determine the type and means by which the system's context is being determined. Therefore this layer reviews all various context types and hardware types (in the form of sensors).

The context determination layer focuses on the following aspects:

- Type of context: What type of context was checked or type of context to which the system adapted. towards

- Type of sensor: What are the various types of sensors being used by the device to determine context.

This section will break down each of the two aforementioned aspects and reviewed for the papers within the study.

\section{$\underline{\text { Type of context }}$}

There are several types of context that a context aware system may employ to adapt the learning for the user. Fig. 5 displays the number of papers in which one or more type of context is detected and for which the system in the paper is subsequently adapted. In order to further understand context adaptation, a categorization of the different types of context is helpful in an attempt to construct a basic context taxonomy. In addition, knowing the various context types being detected and employed by the various systems may help to understand trends within the field of context-aware learning systems. Fig. 5 describes the main classifications of context type to which the system is adapting from the selected papers. The subsequent sub-sections break down the definition for each type of context investigated in this study.

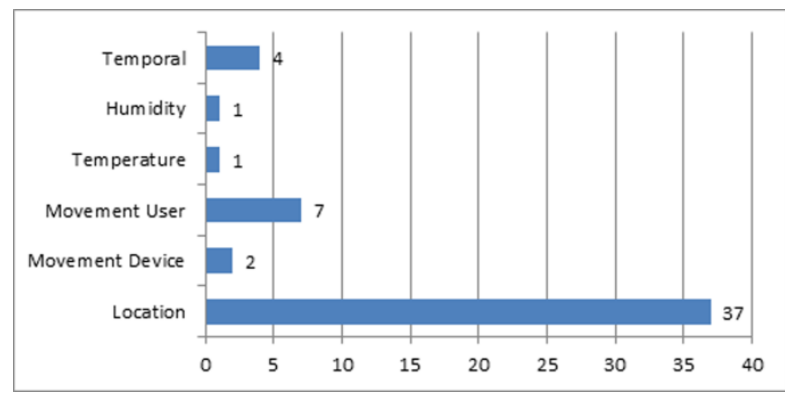

Fig.5. Context Type (Number of Papers)

\section{Ambient: temperature, humidity}

Ambient context-aware adaptivity covers all environmental contexts as they relate to the surrounding area. These are normally absolute in value, and can include a myriad of data types ranging from common everyday sources, to more exotic types common in laboratories. Common everyday ambient context types would include, but are not limited to: temperature, 
luminosity, pressure. More exotic sources may include electromagnetic field detectors or radiation detectors.

\section{Location}

Currently one of the most commonly used context types being queried by systems in this study is location. In this study, location context was analyzed in 37 out of the 41 papers, or $90 \%$. This context refers to the location of the device (and usually the user) on a map. Data results are often given in longitude and latitude, or in the case of RFID, a Boolean indicating whether the device is within range or not. Common sensors used for this type of adaptivity are Global Positioning Systems (GPS) and RFID. This type of adaptivity is triggered by one's location on a map or proximity to a point, regardless of any other data.

\section{Movement}

The context of movement deals with the movement/motion of the device or user. This context was used in 9 out of the 41 papers, for a total of $22 \%$. The movement can be the relative motion between the device and the user, or the relative motion in reference to a $3 \mathrm{rd}$ party. There are two possible scales of motion being discussed, namely the large and the small scale. The large scale is when a device is moving on a scale that can be measured in meters, and typically involves movement around a room, or a town. The small scale of motion, on the other hand, is of motion in the centimeter or smaller range, and involves detecting shaking and jostling of the device. The papers studied in this research identified the two types of movements, that of the device and of the user, for different purposes:

Movement of device

The device may not be attached to the user, and would be classified as movement independent of the user, but more than likely movement relative to the user (small or large scale). The movement of the device context was used in 2 out of the 41 papers, or $5 \%$ of the total.

Movement of user

In this case, both the device and the user would be moving (thus no relative motion to one another). For the purposes of this type of motion, the user and device can be seen as a single entity (small or large scale). The movement of the user context was used in 7 out of the 41 papers, or $17 \%$ of the total.

\section{Temporal}

As the name suggests, temporal context-aware adaptivity adapts to time, either in duration or predetermined times. Temporal context was detected in 4 out of the 41 papers, or $10 \%$.

As can be seen in Fig. 5, the context of location was the most actively used. A possible reason for this is that it is a commonly type of day-to-day context in which we find ourselves. People are forever determining their location (work, home or play), so it is not unreasonable that it would be equally as facile to adapt this type of external context given the availability of the location based sensors. This leads us to the next section, where the types of sensors present are investigated.

\section{Type of sensor}

Mobile devices in the selected papers had either builtin or added sensors to their hardware. In papers such as "The mobile fitness coach: Towards individualized skill assessment using personalized mobile devices" [18], the sensors employed were built into the hardware of the device. On the other hand, in instances such as "A twotier test approach to developing location-aware mobile learning systems for natural science courses" [19], the sensors employed were added separately to the mobile devices. As many different devices come equipped or have the potential to have a myriad of sensors, a breakdown of the sensors used follows and is depicted graphically in Fig 7.

It must be noted that some papers reviewed during the paper selection process, included the use of Quick Response (QR) Codes. QR Codes are two dimensional or square versions of barcodes, and are often found on advertising literature or as part of product marketing. In order to read a QR Code, the user must manually use the camera on the device to read the code. This action is much like taking a photograph, and thus is not an automatic process done by the device and requires specific actions on the part of the user to accomplish. It was decided that due to the nature of the methods that the QR codes were read, they were not automatically adaptive to the context of the device and thus they were not included in this study.

The following three sub-sections describe the commons sensors found in this study. However, future implementations of the framework may include any number of sensors, and should not be limited to the three types mentioned herein.

\section{Accelerometer}

Accelerometers are commonly used for movement detection of both the user and the device. They are designed to detect small scale movements, or of small $\mathrm{G}$ forces. These are found on many modern smartphones, and are accounted for 3 uses in the reviewed papers.

\section{Global positioning system (GPS)}

The GPS receiver or sensor is used for location determination and for movement detection on a much larger scale than an accelerometer. GPS sensors detect/receive signals from geostationary global position satellites, allowing for location determination within +/2 meters. GPS sensors were the second highest ranking and were used within 16 of the 41 , or $39 \%$ of the papers reviewed.

\section{Radio frequency identification (RFID)}

RFID provides a low cost means for small scale location determination. An RFID enabled sensor is active when a suitably equipped reader is within the range of a RFID tag, either active or passive. This type of system is commonly found on keyless entry system in buildings 
and vehicles alike. RFID sensors were used in 23 papers, or $56 \%$ of the papers reviewed.

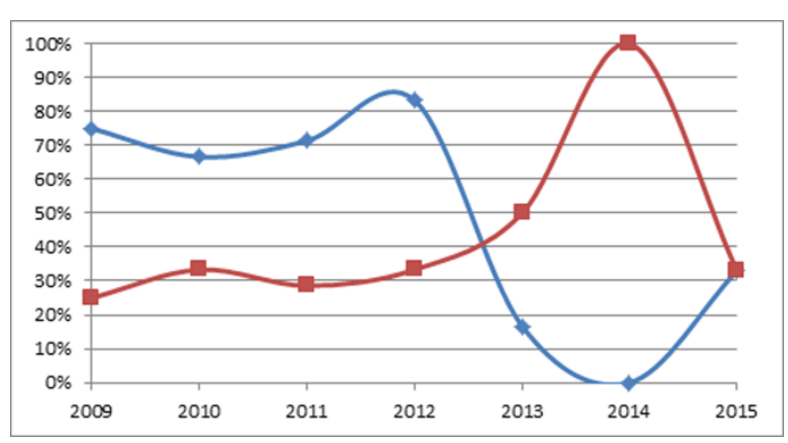

Fig.6. RFID vs GPS Usage (\% of papers published)

As can be seen in Fig. 6, RFID was initially the leader for primary location sensors being used in the studies. However, with the passage of time, and the decrease in the usage of PDAs, as shown in Fig. 2, the trend seems to favour GPS and turn away from RFID based systems.

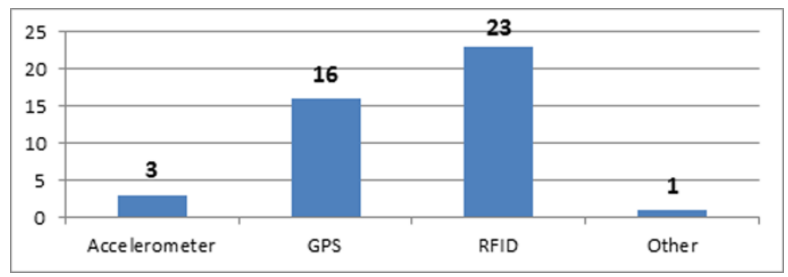

Fig.7. Type of Sensor (Number of Papers)

As shown in Fig. 7, there is an almost prolific use of RFID, followed by GPS, accelerometers, and then others. The reasoning for this seems to relate to the type of context adaptation taking place: adaptation for the location. In Fig. 5, the context of location is used or detected in 37 of the 41 papers.

Although location determination is achieved via the mixture of two types of sensors: GPS and RFID, these two sensors are not exclusively for determining location. In a study by [20], although GPS is used, it does not detect the location, but the rather uses GPS to detect the movement of the user. Therefore, it is to be expected that there may always be a slight difference in the numbers obtained from the analysis of the sensor type versus numbers obtained from the analysis of type of context adaptation. Majority of the papers reviewed utilize a single sensor type. However in contrast, the paper "A context-aware ubiquitous learning environment for conducting complex science experiments" [9] uses four different types of sensors for adaptivity: RFID (for location), temperature, humidity and temporal.

\section{Evaluation layer}

This final layer of the framework addresses the methods by which the studies were evaluated and the participants of the studies as well as the subject matter of the learning being investigated. The components of the evaluation layer are as follows:
- System Evaluation - How the System was evaluated.

- Duration of Testing - How long the device was used for in the study.

- Number of Participants - How many participants were involved.

- Participant Age - Age of the participants.

- Subject Matter - Subject matter, learning type (formal or informal) and discipline.

\section{System evaluation}

There are numerous ways to evaluate the effectiveness or user satisfaction of a system; however, in order to grasp the overall preferred methods, four basic groups/categories were determined to be the most prominent found within the papers analyzed in this study:

- Survey/Questionnaire

- Pre-Post tests

- Interview

- Additional evaluation methods

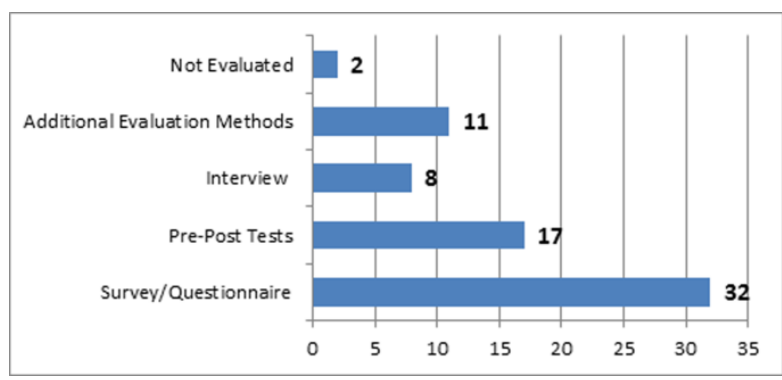

Fig.8. Evaluation Methods (Number of Papers)

The four main types of evaluation method of the studies are shown in Fig. 8. It is worth noting that several papers included more than one evaluation method and thus, the total value is greater than 41. Additionally, there were two papers that did not include evaluation, as they presented only the functional proof of concept. What follows is a breakdown of each type of evaluation method reviewed in this framework.

\section{Survey/questionnaire}

Surveys and questionnaires were the most commonly used evaluation methods comprising 32 of the 41 papers or $78 \%$. Many of the surveys administered in the papers were done to evaluate the effectiveness and general usability of the device and quality of the user experience. These types of evaluations are inexpensive, and can be done en-mass and produce quantitative result suitable for statistical analysis.

\section{Pre-post tests}

The pre-post tests were the tests administered to the participants before and after the study. The participants would be given a test prior to the usage of the contextaware system to assess the participants' knowledge level on a subject. A similar test would be administered after the usage of the device and any improvement would be 
associated with the usage of a device. Under controlled conditions, a control group would be presented which could be used to compare the results (improvement or lack thereof) in score vs. the control group. Much like the survey/questionnaire, this type of evaluation is also inexpensive and is very good at providing quantitative data towards the general success (or failure) of the system as a learning tool. Pre and Post tests were used in 17 out of the 41 papers for a total of $41 \%$.

\section{Interview}

The interview evaluation method would be a face-toface interview usually one-on-one between the participant and the researcher (or proxy), and questions would be asked, usually about the usability and the quality of the learning experience. This is possibly the most time consuming means of evaluation, as it usually requires individual user attention. However, it does provide valuable insights into qualitative aspects of the system being tested that would go beyond the means of the other two methods. Interviews, likely due to their labour intensive nature, were found in only 8 out of the 41 papers for a total of $20 \%$.

\section{Additional evaluation methods}

This section can be used as a general catch-all for evaluation methods that did not fit within the confines of the other three evaluation types. This type of evaluation could include course marks or an in-game scoring assessment, as is found in [21]. Additional evaluation methods were used by a total of 11 papers, or $27 \%$.

\section{Duration of testing}

There was a variance in the amount of time each context-aware system was used in various papers. There were two main divisions noted: the context-aware system was used for the entire course/learning and the contextaware system was used for a part of the course/learning.

The use of the context-aware system for the full course/learning denotes that the entirety of the learning objective was fulfilled using the context-aware system. On the other hand, use of the context-aware system for a part of the course/learning denotes that the context-aware system was used as an aid to the course/learning. In the part course/learning, additional learning was done without the context-aware system.

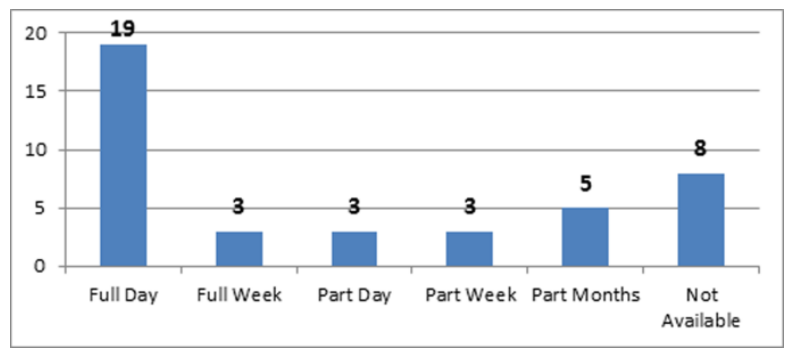

Fig.7. Duration of Testing (Number of Papers)

Fig. 9 shows a tally of the 41 papers reviewed and the duration of testing. The breakdown is as follows:
- Full Day: Indicates the context-aware system was used for the full course/learning and used for the duration of a day to less than a week.

- Full Week: Indicates the context-aware system was used for the full course/learning and used for the duration from 1 or more weeks.

- Part Day: Indicates the context-aware system was used for a part of the course/learning and used for the duration of a day to less than a week.

- Part Week: Indicates the context-aware system was used for a part of the course/learning and used for the duration from 1 or more weeks.

- Part Month: Indicates the context-aware system was used for a part of the course/learning and used for the duration from 1 or more months.

- Not Available: When the duration of the contextaware system's testing or usage was not provided, the Not Available option was selected.

It is interesting to note that the majority of the testing was done in 1 day when comprised of the entire course, or full day. This accounted for a total of 19 out of 41 papers for a total of $46 \%$. Of note is the fact that when the context-aware system was used as part of the course, the duration of the course and length of usage increased. This may be due to the context-aware system being adapted as part of a pre-existing curriculum rather than a custom made course specifically for the context-aware system.

\section{$\underline{\text { Participants }}$}

The number of participants and their ages are an important aspect of any research study. Therefore, this section provides an insight into the varying number of participants of each study along with their age groups.

Fig. 10 shows the number of studies with a given number of participants. Of note is the high number in the 31-60 participant number range, representing, as will later be seen in Fig. 11, the demographic of the participants in the K-12 range: one or more classrooms of students.

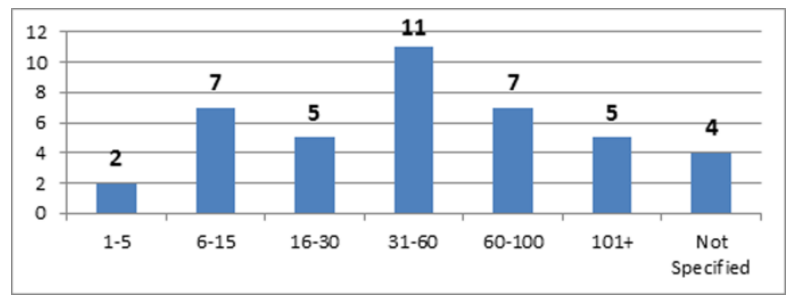

Fig.8. Number Participants in Study (Number of Papers)

Although a combination of several age groups were seen in some papers, the majority of the participants were from the K-12 (Kindergarten to Grade 12, or Age 5 to Age 18) age group (Fig. 11). This age group accounts for 23 out of the 41 papers, or $56 \%$. Out of the $23 \mathrm{~K}-12$ group papers, two of the results were for papers in which the described system did not undergo user evaluation. The large number of papers with participants in K-12 age category may be explained by the advantages of using a 
population sample that is already in a pre-existing learning environment - namely a school. This is true for both K-12 and university aged participants, both representing the two highest participant age groups, with 23 and 12 papers respectively. In both cases, the adaptation of a new learning device/tool may be easier to fit into an existing curriculum and utilizing a captive audience already predisposed to learning.

University students were participants in 12 out of the 41 papers. It must be noted that a division was made between university and adults, as university students may or may not be adults and adults may or may not be university students. Therefore, although cross membership between the groups is possible, it was worthy of note in term of participant age sampling.

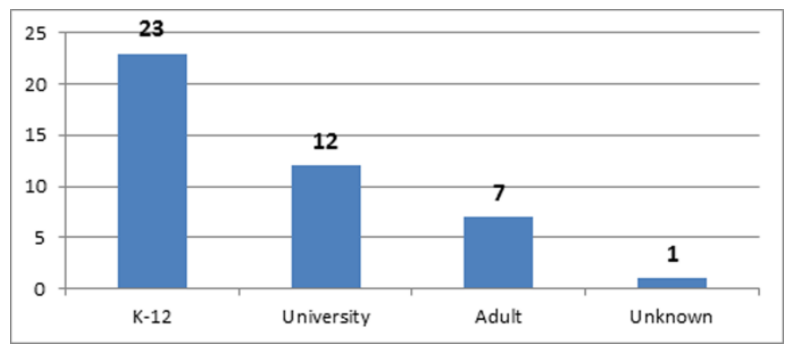

Fig.9. Age Groups of Participants (Number of Papers)

\section{$\underline{\text { Subject matter }}$}

Both the type of learning and the subject matter being taught can be of interest when analyzing various literature. The three main types of learning being tallied in the framework are formal learning, non-formal learning and informal learning [22]. Fig. 12 shows the distribution uncovered after reviewing the 41 papers in this study.

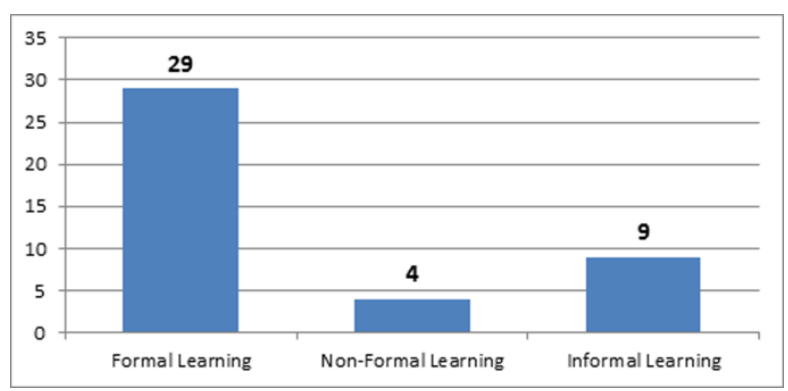

Fig.10. Subject Matter Learning Type (Number of Papers)

The primary learning type focused upon is formal learning, which would certainly agree with the majority of the participant age group (Fig. 11) coming from a formal educational environment where a student/teacher relationship is present. The majority of the subject matter discipline was science, as can be seen in Fig. 13.

There may be several reasons why science, with 21 results, was the predominant subject matter found in the 41 studies. A possible driving factor may be the ease of integration of a science subject matter with the context of the user. As such, many papers which focused on a science-based subject matter as part of their research, focused on environmental/ecological topics such as "An interactive concept map approach to supporting mobile learning activities for natural science courses" [23] and "EcoMOBILE: Integrating augmented reality and probeware with environmental education field trips" [24]. A possible reason for this is that ecology can be descried in terms of location, and may be suitable to describe this particular area of study.

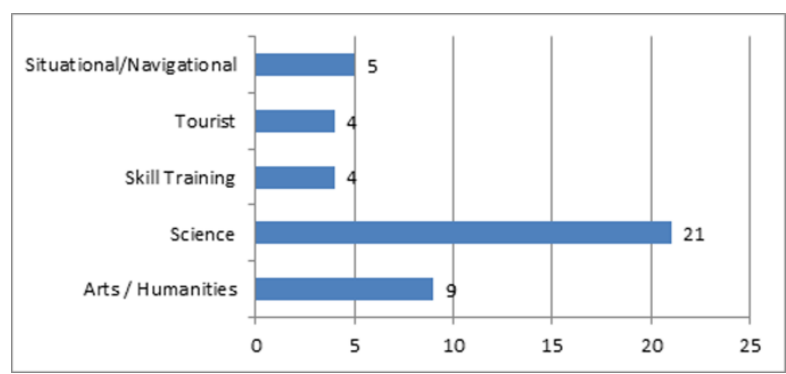

Fig.11. Subject Matter Discipline (Number of Papers)

\section{CONCLUSIONS AND FUTURE WORKS}

Previous literature reviews addressing the field of context-aware mobile learning provided very valuable insights. However, they failed to provide a detailed analysis of the overall direction in terms of hardware, software and populations of the research: key aspects to understanding trends in the field. This study aimed at providing a more comprehensive review of the details of the other studies, both in terms of findings, but also general operational methods and methodologies used. As such, this study was organized according to a framework developed to provide a comprehensive overview on the state of the systems in current research of context-aware mobile learning.

An overall trend presents itself to the reader after reviewing the framework and its contents: context-aware mobile learning is intrinsically tied to, and progresses, with the advancement and ebb and flow of the available hardware. Although there are potentially several options available to researchers, the limiting factor appears to be the system's hardware. This was identified when reviewing the extensive usage of PDAs within the confines of this study. Although, as the constraints imposed on the date range from which to select papers would help explain the usage of rather old PDA technology, the PDAs used were antiquated even for the time of publication. PDAs have fallen out of mainstream usage for the last decade or more, but were still utilized by the researchers. Although the argument can be easily made that as old technology, PDAs were the only financially available solution to the particular study, we suspect it may not be the only reason. It may be the case that the continued usage of the PDA, barring the time delays encountered in publication time, may be due to the ability of the older PDA technology to easily accept the introduction and usage of external sensors. These external sensors provide easier adaptation than those inherent within the factory provided system. Modern day mobile devices have much faster processing power and 
large storage capability than their predecessors, yet it is the sensor data that is key to context-awareness. Therefore, the progression of the research as a whole is arguably entirely dependent upon the technology available to it, and therefore, as is often the case, technology is a limiting factor in progress.

In addition to the technological hurdle of sensor hardware, another factor that is shown by this research is the importance (and perhaps the limitation) of network connectivity. As with the current trends, the usage of offsite server based storage is becoming ubiquitous. In many of the papers reviewed, Wi-Fi tends to be the primary choice for many server-based application setups. This is potentially due to the simple cost factor that cellular data connectivity is much more expensive and also considerably slower than Wi-Fi. However, as Wi-Fi hotspots are becoming more and more ubiquitous, and cellular technology is providing ever faster rates and coverage at an ever reducing cost, that too may change. As these changes take place, one may see an increased usage in server based applications.

Finally, the integration of technology in the learning environment may be of particular interest to researchers: devices were employed for both stand-alone learning systems, and as a component to classes and lectures. Once again, with the ever increasing interconnectivity found in social media, the field may potentially lean towards a greater integration of learning rather than maintaining standalone classes.

It will be very interesting to repeat this study within the next decade, and see if and how the field evolves. The most telling aspect is the decline of the PDA, and thus the possible removal of the ability to utilize RFID tags. How this will change the field, remains to be seen, but given the prevalence of the PDA and RFID hardware in this study, it will likely be quite different within the next 10 years.

\section{ACKNOWLEDGEMENT}

The authors would like to acknowledge NSERC for partial support of this research.

\section{REFERENCES}

[1] Lucke, U. and C. Rensing, A survey on pervasive education. Pervasive and Mobile Computing, 2014. 14: p. 3-16.

[2] Zamzuri, N.H., E.S. Kassim, and M. Shahrom. The Role of Cognitive Styles in Investigating E-learning Usability. in e-Education, e-Business, e-Management, and $e$ Learning, 2010. IC4E'10. International Conference on. 2010. IEEE.

[3] Wong, L.-H. and C.-K. Looi, What seams do we remove in mobile-assisted seamless learning? A critical review of the literature. Computers \& Education, 2011. 57(4): p. 2364-2381.

[4] Lane, N.D., et al., A survey of mobile phone sensing, in Communications Magazine. 2010, IEEE. p. 140-150.

[5] Schilit, B., N. Adams, and R. Want. Context-aware computing applications. in Mobile Computing Systems and Applications, 1994. WMCSA 1994. First Workshop on. 1994. IEEE.
[6] Malek, J., M. Laroussi, and A. Derycke. A multi-layer ubiquitous middleware for bijective adaptation between context and activity in a mobile and collaborative learning. in Systems and Networks Communications, 2006. ICSNC'06. International Conference on. 2006. IEEE.

[7] Weiser, M., R. Gold, and J.S. Brown, The origins of ubiquitous computing research at PARC in the late 1980s. IBM systems journal, 1999. 38(4): p. 693-696.

[8] Cope, B. and M. Kalantzis, Ubiquitous learning: An agenda for educational transformation. Proceedings of the 6th Networked Learning, 2008.

[9] Hwang, G.-J., et al., A context-aware ubiquitous learning environment for conducting complex science experiments. Computers \& Education, 2009. 53(2): p. 402-413.

[10] Yau, J. and M. Joy. A Context-aware and Adaptive Learning Schedule framework for supporting learners' daily routines. in Systems, 2007. ICONS'07. Second International Conference on. 2007. IEEE.

[11] Hwang, G.J. and C.C. Tsai, Research trends in mobile and ubiquitous learning: A review of publications in selected journals from 2001 to 2010. British Journal of Educational Technology, 2011. 42(4): p. E65-E70.

[12] Baldauf, M., S. Dustdar, and F. Rosenberg, A survey on context-aware systems. International Journal of Ad Hoc and Ubiquitous Computing, 2007. 2(4): p. 263-277.

[13] Hong, J.-y., E.-h. Suh, and S.-J. Kim, Context-aware systems: A literature review and classification. Expert Systems with Applications, 2009. 36(4): p. 8509-8522.

[14] Perera, C., et al., Context Aware Computing for The Internet of Things: A Survey. Communications Surveys \& Tutorials, IEEE, 2014. 16(1): p. 414-454.

[15] Wu, H.-K., et al., Current status, opportunities and challenges of augmented reality in education. Computers \& Education, 2013. 62: p. 41-49.

[16] Hwang, G.-J. and P.-H. Wu, Applications, impacts and trends of mobile technology-enhanced learning: a review of 2008-2012 publications in selected SSCI journals. Int. J. Mob. Learn. Organ., 2014. 8(2): p. 83-95.

[17] Santos, P., et al., QuesTInSitu: From tests to routes for assessment in situ activities. Computers \& Education, 2011. 57(4): p. 2517-2534.

[18] Kranz, M., et al., The mobile fitness coach: Towards individualized skill assessment using personalized mobile devices. Pervasive and Mobile Computing, 2013. 9(2): p. 203-215.

[19] Chu, H.-C., et al., A two-tier test approach to developing location-aware mobile learning systems for natural science courses. Computers \& Education, 2010. 55(4): p. 1618-1627.

[20] Buttussi, F. and L. Chittaro, Smarter Phones for Healthier Lifestyles: An Adaptive Fitness Game. Pervasive Computing, IEEE, 2010. 9(4): p. 51-57.

[21] Huizenga, J., et al., Mobile game-based learning in secondary education: engagement, motivation and learning in a mobile city game. Journal of Computer Assisted Learning, 2009. 25(4): p. 332-344.

[22] Dib, C.Z. Formal, non-formal and informal education: Concepts/applicability. in Cooperative Networks in Physics Education-Conference Proceedings. 1988.

[23] Hwang, G.-J., P.-H. Wu, and H.-R. Ke, An interactive concept map approach to supporting mobile learning activities for natural science courses. Computers \& Education, 2011. 57(4): p. 2272-2280.

[24] Kamarainen, A.M., et al., EcoMOBILE: Integrating augmented reality and probeware with environmental education field trips. Computers \& Education, 2013. 68(0): p. 545-556. 


\section{Authors' Profiles}

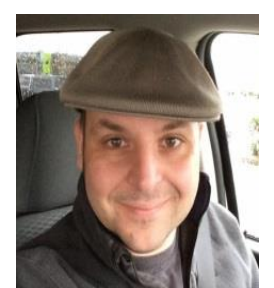

Richard A. W. Tortorella received his MSc degree from Athabasca University, Canada in 2013. He is currently a PhD candidate at the University of Eastern Finland. $\mathrm{He}$ is a member of the IEEE Society and an Assistant Editor for the Smart Learning Environments journal. His current research interests include: context-aware learning systems, m-Learning, and artificial intelligence.

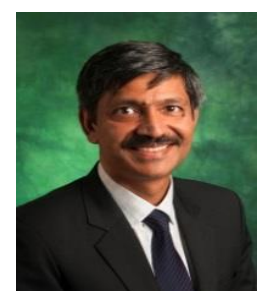

Kinshuk received his $\mathrm{PhD}$ from De Montfort University (Leicester), United Kingdom in 1996. He is the Dean of the College of Information at the University of North Texas. Prior to that, he held the NSERC/CNRL/Xerox/McGraw Hill Research Chair for Adaptivity and Personalization in Informatics, funded by the Federal government of Canada, Provincial government of Alberta, and by national and international industries. He was also Full Professor in the School of Computing and Information Systems and Associate Dean of Faculty of Science and Technology, at Athabasca University, Canada. His research interests include learning analytics; learning technologies; mobile, ubiquitous and location aware learning systems; cognitive profiling; and, interactive technologies.

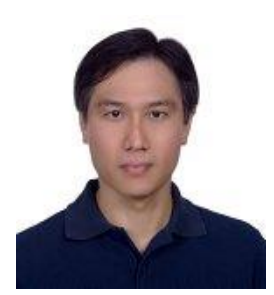

Nian-Shing Chen received his $\mathrm{PhD}$ from the Institute of Computer Science, National Tsing-Hua University, Taiwan in 1990. He is Chair Professor in the Department of Information Management at the National Sun Yat-sen University, Taiwan. He has published over 400 academic papers in the international referred journals, conferences and book chapters. Professor Chen is a senior member of IEEE, ACM. He is Editor-In-Chief of the SSCI-indexed Journal of Educational Technology \& Society. His research interests include assessing e-Learning course performance; online synchronous teaching \& learning; mobile \& ubiquitous learning; embodied cognition \& gamebased learning.

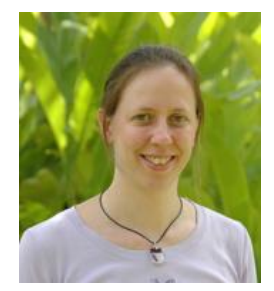

Sabine Graf received her $\mathrm{PhD}$ from Vienna University of Technology, Austria, in 2007. She is presently an Associate Professor at Athabasca University, School of Computing and Information Systems, in Canada. She has published more than 120 peer-reviewed journal papers, book chapters, and conference papers which have been cited over 3,000 times and four conference papers were awarded with a best paper award. Dr Graf is Executive Board Member of the IEEE Technical Committee on Learning Technologies, Editor of the Bulletin of the IEEE Technical Committee on Learning Technology, and Associate Editor of the International Journal of Interaction Design and Architectures. Her research aims at making information systems, especially learning systems, more personalized, intelligent and adaptive. Her research expertise and interests include adaptivity and personalization, student modeling, ubiquitous and mobile learning, artificial intelligence, and learning analytics.

How to cite this paper: Richard A.W. Tortorella, Kinshuk, Nian-Shing Chen, Sabine Graf,"A Classification Framework for Context-aware Mobile Learning Systems", International Journal of Modern Education and Computer Science(IJMECS), Vol.9, No.7, pp.1-11, 2017.DOI: 10.5815/ijmecs.2017.07.01 